\title{
Gestational Diabetes Mellitus: New Diagnostic Criteria
}

\author{
Letícia Nascimento Medeiros Bortolon1, Luciana de Paula Leão Triz¹, \\ Bruna de Souza Faustino', Larissa Bianca Cunha de Sá1, \\ Denise Rosso Tenório Wanderley Rocha1, Alberto Krayyem Arbex ${ }^{1,2}$ \\ ${ }^{1}$ Division of Endocrinology, IPEMED Medical School, São Paulo, Brazil \\ ${ }^{2}$ Visiting Scholar, Harvard T. H. Chan School of Public Health, Harvard University, Boston, USA \\ Email: pauloh-leticia@hotmail.com
}

Received 29 November 2015; accepted 11 January 2016; published 14 January 2016

Copyright (C) 2016 by authors and Scientific Research Publishing Inc.

This work is licensed under the Creative Commons Attribution International License (CC BY). http://creativecommons.org/licenses/by/4.0/

(c) (i) Open Access

\begin{abstract}
Gestational mellitus diabetes (GDM) is a highly prevalent metabolic disorder among pregnant women nowadays. It is defined as any level of glucose intolerance, appearing or first being recognized during pregnancy. It is essential to diagnose and treat GDM early, in order to reduce or avoid complications for mother and fetus. Recently, new guidelines have changed the diagnosis criteria, and it is expected that the prevalence of GDM will increase by approximately $18 \%$. A relevant goal of these new definitions is to provide a better care for pregnant women, in an attempt to reduce fetal and maternal complications. These new criteria will also increase the impact on costs of the health care system. Treatment must be individualized for best results, including a specific diet, physical activity and the use of medications. Metformin and Insulin use are analyzed in detail, in face of new evidences regarding their safety and efficacy during pregnancy.
\end{abstract}

\section{Keywords}

Gestational Diabetes Mellitus, Pregnancy, Glucose Intolerance, Metformin, Insulin, Complications

\section{Introduction}

Gestational diabetes mellitus (GDM) is defined as any degree of glucose intolerance starting with pregnancy or noticed during pregnancy. If the glucose tolerance is not noticed before pregnancy, we call it preexisting diabetes mellitus [1].

The gestational diabetes mellitus is a common metabolic problem, considered an important issue. For this reason, the diagnostics is extremely important [2]. 
The prevalence of GDM is debatable, because it is variable worldwide, depending on the population, human race and the diagnostic criteria defined by each country. International Diabetes Federation estimates that $16 \%$ of the children born alive in 2013 all around the world had complications due to hyperglycemia during pregnancy. It is believed that this prevalence will increase because of the growth of risk factors, mainly obesity and lifestyle [3]. Approximately $90 \%$ of the diabetes cases in pregnant women are considered GDM [4].

The prevalence of GDM is higher in Asian, Latin-American and Indian women [1].

\section{Pathophysiology}

During pregnancy, the maternal tissues become insensitive to insulin. It occurs due to the placental lactogen hormone and other hormones, such as progesterone, cortisol and growth hormone. When the pancreas is unable to offer an appropriate response of insulin to compensate normal insulin resistance, GDM is present. The resistance to insulin leads to maternal hyperglycemia, and this stimulates the fetal hyperinsulinemia [1] [3].

Insulin secretion increases at the beginning of pregnancy, whereas the sensitivity to insulin remains unchanged. At around 20 weeks of pregnancy, insulin sensitivity reduces progressively and it is even lower in the third trimester.

However, after birth, the GMD disappears almost immediately [1]. There are several risk factors for the development of GDM and its identification is extremely important because the sooner the risk factors are identified, the earlier pregnant women are treated in order to implement diagnosis and, thus, minimize complications caused by the disease. The risk factors are: obesity, increased maternal age, previous occurrence of GDM, family history of diabetes, polycystic ovary syndrome, persistent glycosuria, pregnancy-induced hypertension, history of recurrent miscarriage, unexplained fetal death history, macrosomia [1].

\section{Risk Factors}

Screening of GDM is important because it aims to identify women who are at high risks to develop the disease, in order to reduce or avoid risks to maternal and fetal health [3]. Screening for GDM is carried out by identifying the risk factors.

The parameters that determine the greatest risk to disease are: previous GDM, family history of diabetes mellitus, overweight or obesity, increased maternal age. Given these factors, the woman is required to fasting blood glucose, which should be held in early pregnancy [2] [3].

\section{Diagnosis}

The screening of GDM may be initiated during the first prenatal consultation. When the result of fasting glucose is higher than or equal to $126 \mathrm{mg} / \mathrm{dL}$, or random blood glucose levels are higher than or equal to $200 \mathrm{mg} / \mathrm{dL}$ or glycated hemoglobin higher than or equal 6.5\%, the diagnosis of pre-existing diabetes is confirmed. However, when fasting glucose is greater than $92 \mathrm{mg} / \mathrm{dL}$ and lower than $126 \mathrm{mg} / \mathrm{dL}$, GDM is diagnosed, at any gestational age [1].

The main reason for the dilemma of GDM diagnostic criteria is the large number of procedures and different amounts of glucose administered in oral glucose tolerance test [5].

Faced with the various diagnostic parameters used worldwide and the lack of standardization, in 2014, ADA (American Diabetes Association) recommends new criteria for diagnosis. Pregnant women in early pregnancy who do not meet the diagnostic criteria for GDM should be retested between $24^{\text {th }}$ and $28^{\text {th }}$ gestational weeks. A single positive test is enough for the diagnosis, according to the information shown in Table 1 [6].

With these new diagnostic criteria, it is estimated that the prevalence of GDM will increase by approximately $18 \%$, thus generating a greater impact on costs in the health system and better care for pregnant women in an attempt to reduce fetal and maternal complications caused by disease [3].

Table 1. Diagnostic criteria for overt diabetes and gestational diabetes using a 2-hour 75-g OGTT at 24 to 28 weeks gestational.

\begin{tabular}{|c|c|c|c|}
\hline Diagnosis & Fasting Plasma Glucose, mg/dL & 1-h Value, mg/dL & 2-h Value, mg/dL \\
\hline Overt diabetes (type 1 , type 2 , or other) & $\geq 126 \mathrm{mg} / \mathrm{dL}$ & Not applicable. & $\geq 200 \mathrm{mg} / \mathrm{dL}$ \\
\hline Gestational diabetes & $\geq 92 \mathrm{mg} / \mathrm{dL}$ & $\geq 180 \mathrm{mg} / \mathrm{dL}$ & $\geq 153 \mathrm{mg} / \mathrm{dL}$ \\
\hline
\end{tabular}




\section{Complications}

Complications related to the GDM affect both maternal and fetal health and thereafter child health.

After birth, the hypoglycemic effects of placental hormones are eliminated quickly, so the vast majority of pregnant women return to their pre-pregnancy glycemic state, i.e., after removal of the placenta, the insulin resistant state that is characterized in pregnancy and insulin requirements fall dramatically [7] [8].

A large majority of pregnant women return to normoglycemia after birth, as follows:

- $2 / 3$ will have gestational diabetes mellitus again in a next pregnancy;

- $20 \%$ have impaired glucose tolerance in the postpartum period;

- $50 \%$ will have diabetes mellitus type 2 [9].

Women who have had GDM have a significant higher risk to develop type 2 diabetes mellitus. These women must be accompanied with oral glucose tolerance test (OGTT) from 2 to 3 months after childbirth, and they must receive annual monitoring with OGTT and yearly be tested for fasting glucose [3].

The most common fetal complications are macrosomia, spontaneous abortion, congenital malformation, intrauterine death.

The most common maternal complications are: risk of diabetes mellitus recurrence in future pregnancies, future possibility of diabetes mellitus, polyhydramnios, pregnancy toxaemia, urinary tract infection, candidiasis, higher incidence of premature childbirth, cesarean delivery.

The most common neonatal complications include hypoglycemia, hypocalcemia, polycythemia, jaundice requiring phototherapy, trauma (shoulder dystocia) and respiratory distress syndrome.

Gestational diabetes mellitus is not an absolute indication of cesarean birth and the birth via is an obstetric decision. Pregnant women who have great metabolic control and who have no obstetric history of perinatal death, macrosomia or associated complications such as hypertension and preeclampsia can wait for the spontaneous evolution of delivery [10].

It is advised to indicate scheduled cesarean birth for all pregnant women presenting fetal weight $\geq 4500$ grams, knowing that the greater the weight of the fetus, the greater the incidence of complications if a vaginal birth is performed. The most common complications due to weight are: shoulder dystocia injuries and permanent brachial plexus [7].

\section{Treatment and Prevention}

The aim of the treatment is to maintain normal blood glucose levels through pregnancy, and avoid periods of hypoglycemia and hyperglycemia. The main therapeutic goal throughout pregnancy is to avoid the adverse events caused by hyperglycemia [6] [11].

The GDM treatment is set according to the needs of each patient, including nutritional counseling and pharmacological treatment [12].

A diet with the intake of dietary fiber, mainly cereals and fruit reduce the risk of developing GDM. Each $5 \mathrm{~g}$ of fruit and cereal fiber decreases the risk of GDM at a range of $23 \%$ and $26 \%$. The intake of dietary fiber reduces appetite and thus reduces the total energy consumption, delays gastric emptying, and decreases glucose uptake. The ideal diet is the one that includes chicken, fish, fruits, vegetables and green leaves [13].

Exercise has important role on glycemic control and thus may prevent, reduce or delay the need for insulin. Exercise is responsible for improving glycemic control in women with GDM, but also plays an important role on preventing the development of GDM. There is not a specific kind on exercise recommended. However moderate intensity exercises are indicated, but even low-intensity exercise has shown benefits in mood, balance, back pain and urinary incontinence [3].

The frequency to carry out the exercises should be increased gradually and it is suggested $45 \mathrm{~min} /$ day, 5 times a week [14].

There are some studies that show a possible reduced incidence of GDM with the use of probiotics. The most commonly used are Lactobacillus rhamnosus and Bifidobacterium lactis [13]. Probiotics and vitamin D are being subject of studies regarding GDM prevention or treatment, without any evidence of efficacy so far [13].

Vitamin D deficiency is a global problem and is associated with cardiovascular disease, musculoskeletal diseases, and autoimmune diseases. Studies on the association of vitamin D deficiency and higher incidence of GDM have been carried out. Vitamin D at normal levels (higher than $30 \mathrm{ng} / \mathrm{ml}$ ) is associated with a lower insulin resistance [13]. 
After GDM diagnosis, all patients should receive directions on the diet and the physical exercises. It is estimated that $70 \%$ to $85 \%$ of cases of GDM can be controlled with changes in the lifestyle. If aim goal is not achieved within 2 weeks, pharmacotherapy should be started. The ideal glycemic control according to the information is shown in Table 2 [1] [4] [6].

\subsection{Insulin Use in Gestational Diabetes}

Insulin is the first-line therapy for treatment of GDM according to most Guidelines, as insulin does not cross the placental barrier, providing safety to the fetus [4].

Fetal growth criteria may also be used to indicate the beginning of the use of insulin. This is an option indicated by Buchanan, in which the fetal abdominal circumference is measured and when it is at or above the $75^{\text {th }}$ percentile on an ultrasound scan performed between $29^{\text {th }}$ and $33^{\text {rd }}$ week of pregnancy, the use of insulin is recommended [15].

The choice of which insulin is used depends on the hyperglycemia pattern noticed during glycemic control. If there is prevalence of fasting or preprandial hyperglycemia, it is indicated to use a long-acting insulin. If there is a predominance of postprandial hyperglycemia, rapid insulin is suggested [16].

Optimization of insulin treatment should be constant with frequent dose adjustments, which should be based on daily monitoring of blood glucose. The insulin needs may vary according to the gestation period. In the first quarter, in general, the demand for insulin is lower, in the second and third quarters higher doses of insulin are used [17].

For patients who are resistant to use insulin, an alternative is the use of metformin and glibenclamide. The use of them is regarded as B category, as they are considered safe and effective, but the long-term safety data are not yet available [4].

Glibenclamide is a second-generation sulfonylurea, which acts by binding to the pancreatic beta cell receptors, blocking the potassium channel and opening calcium channels, stimulating insulin release. As it reduces hepatic glucose production, it contributes to fasting hyperglycemia. The transfer of glibenclamide by the placenta barrier exists, but it is minor. The causes of this low transfer are suggested by Koren, citing that the drug binding to lipoproteins is $99 \%$, and only $0.2 \%$ would be free to cross the placental lye, with the half life for rapid elimination, about 4 hours, fact that explains the non-detection of the drug in fetal blood soon after birth [17].

It is believed that glibenclamide is an acceptable alternative for pregnant women who cannot control blood glucose with diet and oppose the use of insulin. Its use during pregnancy also raises concerns on security issue, so studies are still being conducted to research on the side effects during pregnancy. There is still nothing concrete regarding glibenclamide, studies have shown that glibenclamide is not associated with risk of neonatal hypoglycemia or congenital anomalies, but studies are still being carried out and if the decision is for the prescription of the drug, the pregnant woman needs to be instructed on the uncertainties [18].

\subsection{Metformin Use in Pregnancy}

Metformin is the drug chosen for treating type 2 diabetes in patients who are not pregnant, and appears to be a great oral hypoglycemic agent for the treatment of GDM. Metformin acts by decreasing hepatic gluconeogenesis and enhancing peripheral absorption of glucose. Moreover, their effect is beneficial by not showing weight gain and not causing hypoglycemia [17].

The use of metformin in pregnant women is still controversial in Guidelines, but there are increasing strong evidences on the effectiveness of metformin during pregnancy, and it has been suggested that metformin is a safe alternative both in monotherapy and associated with the use of insulin [12].

Metformin exhibits beneficial effects for the treatment of patients who have gestational diabetes mellitus; it appears to be safe if used in a short period, being effective in many women. However, there is a considerable

Table 2. The ideal glycemic control aims at.

Target Value, $\mathrm{mg} / \mathrm{dL}$

Preprandial blood glucose

$\leq 95 \mathrm{mg} / \mathrm{dL}$

$1 \mathrm{~h}$ after the start of a meal

$\leq 140 \mathrm{mg} / \mathrm{dL}$

$2 \mathrm{~h}$ after the start of a meal

$\leq 120 \mathrm{mg} / \mathrm{dL}$ 
number of women who have unsatisfactory glycemic control with only its use, requiring a combination with insulin. Although metformin has possible effect on crossing the placenta lye, it is not responsible for teratogenic effects during the first pregnancy quarter [17]-[19].

The long-term effects of metformin on the future metabolic disorders of the fetus are still unknown. It is believed that metformin induces a more favorable intrauterine environment (positive "Prenatal Programming") and as a result reduces long-term metabolic complications, such as diabetes mellitus and obesity [12].

In some cases the use of metformin alone is not sufficient to maintain appropriate glycemic control, and the use of insulin is needed. Supplementation with insulin may be needed in almost half of pregnant women and is more common in obese women and fasting hyperglycemia. Pregnant women candidates for the use of metformin are between 18 - 45 years, gestational age between 20 and 33 weeks and lower fasting glucose $140 \mathrm{mg} / \mathrm{dl}$ [20].

\section{Recommendations Based on This Review}

The screening of GDM is accomplished by identifying its risk factors. It should begin during the first prenatal consultation. In the presence of these factors, such patients should undergo a fasting blood glucose testing [1]-[3].

- If the levels of fasting glucose are higher than or equal to $126 \mathrm{mg} / \mathrm{dL}$, or a random blood glucose test level is higher than or equal to $200 \mathrm{mg} / \mathrm{dL}$, or if glycated hemoglobin levels are higher than or equal $6.5 \%$, the diagnosis of pre-existing diabetes is confirmed [1] [6].

- If the result of fasting glucose level is higher than $92 \mathrm{mg} / \mathrm{dL}$ and lower than $126 \mathrm{mg} / \mathrm{dL}$, GDM is diagnosed, at any gestational age [1] [6].

Pregnant women in early pregnancy who do not meet the diagnostic criteria for GDM should be retested between the $24^{\text {th }}$ and $28^{\text {th }}$ gestational weeks. A single positive test is enough for the diagnosis, according to the information bellow [6]:

- Fasting blood glucose higher than or equal than $92 \mathrm{mg} / \mathrm{dL}$.

- 75-g OGTT-Value after 1 hour higher than or equal than $180 \mathrm{mg} / \mathrm{dL}$.

- 75-g OGTT—Value after 2 hour higher than or equal than $153 \mathrm{mg} / \mathrm{dL}$.

The GDM treatment is adjusted according to the needs of each patient [12].

Diets with a high intake of dietary fiber reduce the risk of developing GDM [13]. Exercise has an important role on glycemic control and thus may prevent, reduce or delay the need for insulin. Exercise is responsible for improving glycemic control in women with GDM, but also plays an important role on preventing the development of GDM [14]. Probiotics and vitamin D are being subject of studies regarding GDM prevention or treatment, without any evidence of efficacy so far [13].

In case of failure in improving lifestyle, insulin is the first-line therapy for treatment of GDM, according to most guidelines. Metformin is being increasingly considered as a safe and effective option [4].

Fetal growth criteria may also be used to indicate the beginning of the use of insulin. The fetal abdominal circumference is measured and when it is at or above the $75^{\text {th }}$ percentile on an ultrasound scan performed between $29^{\text {th }}$ and $33^{\text {rd }}$ week of pregnancy, the use of insulin is recommended [15].

The aim of the treatment is to maintain normal blood glucose levels through pregnancy. The ideal glycemic control aims at [1] [4] [6]:

- Preprandial blood glucose lower than or equal than $95 \mathrm{mg} / \mathrm{dL}$.

- $1 \mathrm{~h}$ after the start of a meal lower than or equal than $140 \mathrm{mg} / \mathrm{dL}$.

- $2 \mathrm{~h}$ after the start of a meal lower than or equal than $120 \mathrm{mg} / \mathrm{dL}$.

GDM is not an absolute indication of cesarean delivery and the way of delivery is an obstetric decision.

Women who have had GDM have a significant higher risk to develop type 2 diabetes mellitus. These women must be accompanied with oral glucose tolerance test (OGTT) from 2 to 3 months after childbirth, and they must receive annual monitoring with OGTT and yearly be tested for fasting glucose [3].

\section{Conclusions}

Gestational diabetes mellitus is a disease with serious consequences to the mother and the fetus, showing some irreversible complications on metabolism. This disease is frequent in the clinical practice due to the high prevalence of overweight and obesity as associated diseases, along with an inadequate diet and a sedentary lifestyle.

During screening and diagnosis of the disease, it is very important to recognize the correct diabetes type in- 
volved, defining whether it is a true gestational diabetes mellitus or a pre-existing mellitus diabetes, diagnosed only in pregnancy.

Hyperglycemia is responsible for the alterations on mother and fetus metabolism, but pre-existing diabetes has an even greater risk for pregnancy, because the glycemic alterations are already present since the first 12 weeks, during the organogenesis process of the fetus.

New diagnosis criteria have increased the prevalence of GDM, and intend to prevent complications and offer a better glycemic control.

The treatment of gestational diabetes mellitus must start as soon as the diagnosis is established. The nutritional intervention and the treatment with medications have an important role on lowering the complications rate and, therefore, food guidance must always be indicated and quickly started. Insulin and Metformin are good alternatives available for controlling glucose levels and avoiding potential complications to mother and newborn.

\section{Acknowledgements}

The authors would like to thank IPEMED Brazil for continuous support on medical research. We also thank Prof. Dr. Aline Marcadenti for her unique dedication to research.

\section{References}

[1] Alfadhli, E.M. (2015) Gestational Diabetes Mellitus. Saudi Medical Journal, 36, 399-406. http://dx.doi.org/10.15537/smj.2015.4.10307

[2] Mukesh, M.A. (2015) Gestational Diabetes Mellitus: Na Update on the Current International Diagnostic Criteria. World Journal of Diabetes, 6, 782-791.

[3] Kampmann, U., et al. (2015) Gestational Diabetes: A Clinical Update. World Journal of Diabetes, 6, 1065-1072. http://dx.doi.org/10.4239/wjd.v6.i8.1065

[4] Kelley, K.W., Carroll, D.G. and Meyer, A. (2015) A Review of Current Treatment Strategies for Gestational Diabetes Mellitus. Drugs in Context, 4, Article ID: 212282.

[5] Gupta, Y., Kalra B., Baruah, M.P., Singla, R. and Kalra, S. (2015) Update Guidelines on Screening for Diabetes. International Journal of Women's Health, 7, 539-550.

[6] Blumer, I., et al. (2013) Diabetes and Pregnancy: An Endocrine Society Clinical Practice Guideline. Journal of Clinical Endocrinology \& Metabolism, 98, 4227-4249. http://dx.doi.org/10.1210/jc.2013-2465

[7] Caughey, A.B. (2014) Gestational Diabetes Mellitus: Obstetrical Issues and Management. http://www.uptodate.com/contents/gestational-diabetes-mellitus-obstetrical-issues-and-management

[8] Kjos, S.L., et al. (2014) Pregestational and Gestational Diabetes: Intrapartum and Postpartum Glycemic Control. http://www.uptodate.com/contents/pregestational-and-gestational-diabetes-intrapartum-and-postpartum-glycemic-contr ol

[9] Stasenko, M., et al. (2010) Postpartum Follow-Up for Women with Gestational Diabetes Mellitus. American Journal of Perinatology, 27, 737-742. http://dx.doi.org/10.1055/s-0030-1253557

[10] Campos, M.A., Fagundes, A. and Seligman, L.C. (2002) Manejo e diagnóstico do diabetes mellitus gestacional. Momento \& Perspectivas em Saúde, 15, 34-39.

[11] Pérez, O., Saba, T., Padrón, M.A. and Molina V, R. (2012) Diabetes Mellitus Gestacional. Revista Venezolana de Endocrinologia y Metabolismo, 10, 22-33.

[12] Marques, P., Carvalho, M.R., Pinto, L. and Guerra, S. (2014) Metformin Safety in the Management of Gestational Diabetes. Endocrine Practice, 20, 1022-1031. http://www.ncbi.nlm.nih.gov/pubmed http://dx.doi.org/10.4158/EP14018.OR

[13] Gunasekaran, P.K. (2015) Changing Trends in Management of Gestational Diabetes Mellitus. World Journal of Diabetes, 6, 284-295. http://dx.doi.org/10.4239/wjd.v6.i2.284

[14] Padatachee, C. and Coombes, J. (2015) Exercise Guidelines for Gestational Diabetes Mellitus. World Journal of Diabetes, 6, 1033-1044. http://dx.doi.org/10.4239/wjd.v6.i8.1033

[15] Diretrizes da Sociedade Brasileira de Diabetes (2013/2014), 184-188.

[16] Jacob, T.A., et al. (2014) Diabetes Mellitus Gestacional: Uma revisão de literatura. Brazilian Journal of Surgery and Clinical Research, 6, 33-37.

[17] Silva, J.C., Narciso, D.R.R., Coral, M.L., Caetano, T., Janeczko, L. and Bertini, A.M. (2012) Hipoglicemiantes Orais versus Insulinoterapia no Tratamento do Diabetes Gestacional. Arquivos Catarinenses de Medicina, 41, 72-77. 
[18] Singh, S.R., Ahmad, F., Lal, A., Yu, C., Bai, Z. and Bennett, H. (2009) Efficacy and Safety of Insulin Analogues for the Management of Diabetes Mellitus: A Meta-Analysis. CMAJ, 180, 385-397. http://dx.doi.org/10.1503/cmaj.081041

[19] Poolsup, N., Suksomboonn, N. and Amin, M. (2014) Efficacy and Safety of Oral Antidiabetic Drugs in Comparison to Insulin in Treating Gestational Diabetes Mellitus: A Meta-Analysis. PLoS ONE, 9, e109985.

http://dx.doi.org/10.1371/journal.pone.0109985

[20] Riskin, A.G. (2014) Infanty of a Diabetic Mother. http://www.uptodate.com/contents/infant-of-a-diabetic-mother 